\title{
DYNAMICS OF AGB STARS AND PLANETARY NEBULAE IN \\ THE GALAXY
}

\author{
H. DEJONGHE \\ Sterrenkundig Observatorium, Universiteit Gent, Belgium
}

\begin{abstract}
The available kinematical data on $\mathrm{OH} / \mathrm{IR}$ stars and $\mathrm{PNs}$ are reviewed. Dynamical models for the OH/IR stars are presented.
\end{abstract}

Key words: Dynamics - Equilibrium models - the Galaxy

\section{Introduction}

It is proverbial that one picture says more than a thousand words. Pictures shape our ideas about all kinds of astronomical objects, from planets to the universe itself. Moreover, the inquiring mind transcends the (visual) appearance of the things with theory, only to find itself trying to visualise abstract concepts back again (graphs,...). A good example thereof is the H-R diagram, which is a picture that compactly summarizes stellar evolution.

In this contribution, I will try to introduce a particular way of visualising the dynamical state of a galaxy. This doesn't seem to fit in well with the subject of this symposium, were it not that I will use tracer populations of AGB stars (mainly $\mathrm{OH} / \mathrm{IR}$ stars and PNs) to reach that goal.

\section{Elements of Dynamical Modelling}

The ultimate dynamical knowledge is to know for every star, at all times, its location in phase space, which is the product of configuration space (normal 3-space) and velocity space. On the other hand, such a complete knowledge is impossible and (as of now) unmanageable; this is why both theory and assumptions are needed to simplify this ultimate picture.

When studying the dynamics of a tracer population, it is obvious that selfconsistency is not required, i.e. the gravitational potential is not generated by the tracer population. This means that the gravitational potential can be decoupled from the original set of equations, and this potential therefore must be a given. The specification of the gravitational potential is the first important decision one has to make when building an equilibrium model. In particular, one has to decide on the prevailing geometry, i.e. whether the potential is spherical, axisymmetric or triaxial.

The potential generates structure in phase space, because it creates orbits. The quintessential orbit is the linear harmonic oscillator, e.g. a spring. At every moment it has a length $z$ which changes at a rate $v_{z}$. Phase space is the 2-dimensional space $\left(z, v_{z}\right)$. If we do not know the dynamical state of the spring completely, it is natural to ask what information we can single out as particularly important. The maximum length $z_{m}$ must be such a quantity, because then, at least, we can confine the length of the spring, though we've lost the ability to predict its actual length 
at any particular moment. This $z_{m}$ is an example of an integral of the motion ${ }^{1}$ : it is a function of phase space coordinates that remains a constant along the orbit, and therefore it can be used as a label for that orbit. Hence, we can now describe the linear harmonic oscillator with $z_{m}$ ( a constant) and only one rapidly changing coordinate $\left(z\right.$, or $v_{z}$, or something else).

In 3 dimensions, one would expect 3 constants of the motion and 3 rapidly changing variables for every orbit. This is true for integrable potentials, by definition. Most potentials however are not integrable, but it is likely that for most astrophysical purposes there exist good integrable fits (Goodman and Schwarzschild 1981, Dejonghe \& de Zeeuw 1988), safe possibly for tumbling triaxial figures. A very elegant class of integrable potentials are the Stäckel potentials, which have the nice property that the integrals of the motion are quadratic functions of the velocities.

In order to better understand the significance of these integrals, let's consider the following experiment. We affix many springs to a flat surface. The springs only vibrate in the $z$-direction (perpendicular to the surface), and hence their $x$ and $y$ coordinates which are markers on the surface are constants of the motion. Now we disturb the springs, for example by pushing them down simultaneously by hand. The imprint of the hand will be lost very quickly, and in the analysis of the resulting dynamical state, it will certainly not matter very much to focus on the description of the rapidly changing coordinates. If we only knew $z_{m}(x, y)$, then we would know the profile of the perturber, which is everything that there is to know in this experiment. Consider next the somewhat different situation that at every location $(x, y)$ there are a lot of springs (for example molecules), which may or may not start to vibrate due to infalling light, then the number of excitations $N$ will be proportional with the intensity, while the degree of excitation (the $z_{m}$ ) will tell us something about the wavelength of the infalling light. The function $N\left(x, y, z_{m}\right)$ we call a distribution function. It is written here as a function in integral space. It cannot exist without a medium for which it is a probability density, though it may provide us with important information on something else (the infalling light). This function is very analogous to the concept with the same name in stellar dynamics; the medium there is called a tracer population. On a photographic plate, the distribution function is a faithful representation of the perturbing radiation, and in stellar dynamics it is hoped that the distribution function will teach us similarly important things about the formation of galaxies.

Though the distribution function $F$ is defined as a probability density in phase space, we will, according to Jeans' theorem, write it as a function of the integrals of the motion. Hence, it is, for all purposes, a function in integral space. For time independent potentials, the specific binding energy $E$ is always one of them, and according to the geometry and the potential, there may be 2 more integrals. The big difference with the previous examples is that all these integrals are highly non-local, just as an orbit is.

In order to determine this distribution function, we must write down its relation with observable quantities. This relation can almost always be written in the form

1 The concept is actually rather complicated, and a precise definition is far beyond the scope of this contribution 
of an average of the distribution function. As already indicated, the body of data can be very inhomogeneous, including star counts, mean velocities and velocity dispersions, line profiles, proper motions, etc... No general theorem exists that would enable us to decide on the uniqueness of this inversion, let alone analytic procedures to perform such an inversion. Hence, a pragmatic approach is in order.

One way to proceed is with quadratic programming (QP, Dejonghe 1989). In this method we assume that the distribution function can be written as a linear combination of (preferably analytically simple) components, with coefficients $c_{i}$. A $\chi^{2}$-type function (quadratic in the $c_{i}$ ) is then mimimized, subject to the constraint that the distribution function must be positive everywhere (linear constraints in the $c_{i}$ ).

Only numerical experience at this point can give us an idea to what degree we can have confidence in the computed distribution function. It is obvious that the more the data cover phase space, the more the distribution function will be constrained. Also, the more restrictive we are in the functional form of $F$ (function of one, two, or three integrals), the less indeterminacy we will encounter when trying to determine a distribution function, but also the less realistic our results may be. Only partial results on these issues are available (Dejonghe \& Merritt 1992), and there still remains a lot to do.

\section{Some models for tracer populations}

As an obvious consequence from the preceding section, a tracer population which may be used to stake some claims about the global dynamical state of our Galaxy must be present in a substantial volume of the Calaxy, and must also be detectable there. This inevitably puts infrared astronomy in a privileged place, and the IRAS satellite certainly has earned her marks in that respect.

\subsection{OH/IR STARS}

The $\mathrm{OH} / \mathrm{IR}$ stars have the advantage of being old, strong infrared emittors with a characteristic spectrum around the $1612 \mathrm{Mhz}$ maser peak. Their mostly old age and AGB status make them rather useful for equilibrium dynamical modelling, since it helps that we can assume that somehow older objects must have undergone some form of relaxation, which is presumably sufficient (but not necessary) for equilibrium. Their strong infrared emission makes them shine right through the dusty galactic plane (GP), a property which is needed for a sufficient spatial coverage. The characteristic double maser peak, caused by an expanding shell, is easily recognizable, and provides a simple way of measuring the line--of-sight velocity. In addition, the velocity of the expanding circumstellar shell may indicate an age, in the (statistical) sense that larger expansion velocities are associated with younger stars. This provides an interesting test on the models, since it can be expected that the younger population is more confined to the disk.

All this is reason enough to systematically search for them, and this has been done by Eder et.al. (1988), Sivagnanam et.al. (1989) and te Lintel Hekkert et.al. (1991a), hereafter tLH, and further references therein. The IRAS PSC provides 
a $F_{\nu}(12 \mu m) / F_{\nu}(25 \mu m)$ versus $F_{\nu}(25 \mu m) / F_{\nu}(60 \mu m)$ color diagram in which the $\mathrm{OH} / \mathrm{IR}$ stars occupy a fairly well defined place. A list of candidate $\mathrm{OH} / \mathrm{IR}$ stars results, and these are then individually radio-checked for the $1612 \mathrm{Mhz}$ emission. About $1500 \mathrm{OH} / \mathrm{IR}$ stars are now known.

The IRAS satellite was severely confused in the GP, because of the high density of sources. In order to find $\mathrm{OH} / \mathrm{IR}$ stars there, one must resort to mapping type surveys. The first such survey close to the galactic center (GC) was done by Habing et.al. (1983), and yielded 34 stars within a radius of $1^{\circ}$ around the GC. This survey has been substantially improved in a more limited region by Lindqvist et.al. (1992a), hereafter L, yielding 134 stars. None of these surveys have been completely satisfactory in their velocity coverage, for technical and feasibility reasons. a window of $\pm 217 \mathrm{~km} / \mathrm{s}$, the tLH sample that (a few) high velocity stars can be expected to turn up when searching for them (van Langevelde 1992).
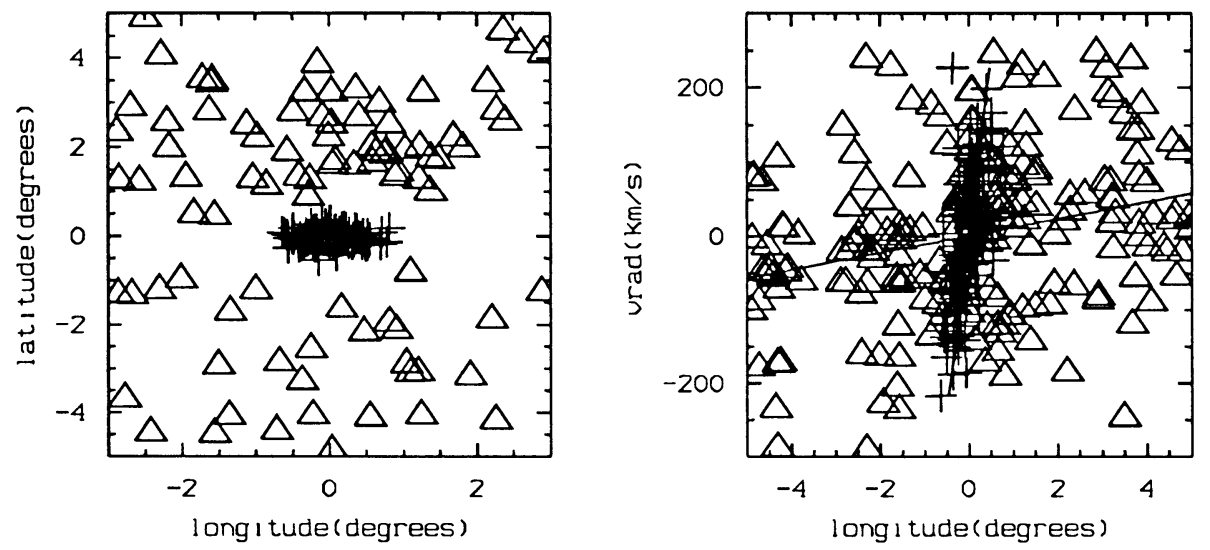

Fig. 1. (a) The L sample (crosses) and the truncated tLH sample (triangles) in $(\ell, b)$ on the sky. (b) The L sample (crosses) and the truncated tLH sample (triangles) in $\left(\ell, v_{r}\right)$ space.

I will now summarize the first results from the tLH and L surveys. Some of these results can be found in te Lintel Hekkert et.al. (1991b), and Lindqvist et.al. (1992b). Figure la shows the $(\ell, b)$ diagrams for the $\mathrm{L}$ and the tLH samples, the latter being truncated to $\pm 3^{\circ}$ in longitude and $\pm 5^{\circ}$ in latitude. It is obvious that both samples are complementary, but certainly not enough so. Currently a consortium headed by Habing and te Lintel Hekkert is working on surveys at the VLA and the AT to fill in a few gaps. The tLH survey is believed to be fairly complete up to $3 \mathrm{Jy}$ at $12 \mu \mathrm{m}$, for $|b|>2.5^{\circ}$, the latter limitation due to IRAS. This is very clear from the "zone of avoidance" in Fig. 1 for the tLH sample. In future surveys, considerable attention and care will have to be taken to treat completeness properly, in order to link the different surveys together.

Figure 1 b shows the $\left(\ell, v_{r}\right)$ plot for both samples (heliocentric velocities). The regression lines are the linear approximations to the rotation curves. The L rotation curve (crosses) in this plot has a slope of about $500 \mathrm{~km} / \mathrm{s} /$ degree or about 3.7 $\mathrm{km} / \mathrm{s} / \mathrm{pc}$, using $R_{(:)}=7.5 \mathrm{kpc}$. These values are about right as is obvious from the 
plot, but are a factor of three higher than quoted by Lindqvist et.al. (1992b). They note however that the slope is very uncertain, depending on the elimination of a few stars, as must be since the slope is very steep. The mass of the point source in the GC is therefore not so well constrained, and values are given in Lindquist et.al. (1992b). The slope of the tLH sample is $11 \mathrm{~km} / \mathrm{s} /$ degree or about $82 \mathrm{~km} / \mathrm{s} / \mathrm{kpc}$. Such a rotation curve reaches its presumed peak value of about $220 \mathrm{~km} / \mathrm{s}$ at about $2.5 \mathrm{kpc}$, which is very reasonable. These slopes are so different because the tLH sample ignores the $\mathrm{GC}$ and the GP.
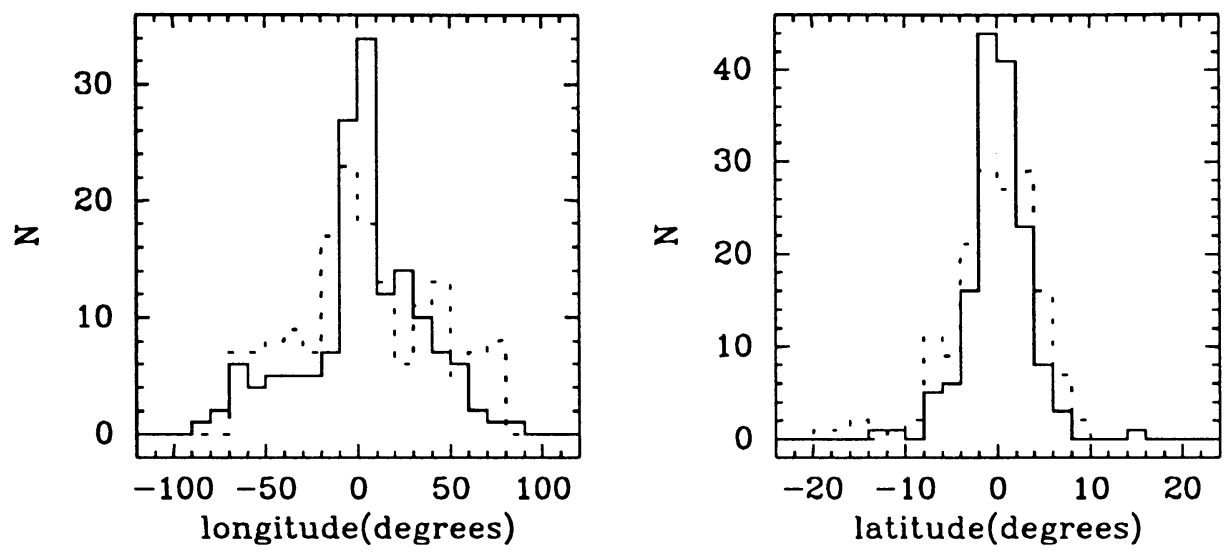

Fig. 2. The histograms of old stars in the tLH sample (dashes) and the young stars (solid) as a function of galactic longitude and latitude

From the analysis by Lindqvist et.al. (1992b) it would seem that indeed the projected velocity dispersion of the younger stars is smaller than the dispersion for the older stars, as one could expect if stars are born on primarily circular orbits. In Fig. 2 we see histograms as a function of longitude and latitude for a selection of the tLH sample, divided into two groups defined by the "oldest" and "youngest" stars, each containing about 150 stars. It is clear that, a few dissenters notwithstanding, the younger population is more confined to the disk, and somewhat more bulgy.

As explained in the first section, equilibrium dynamical modelling needs an assumption on the potential. There is no obvious sign of triaxiality in the tLH data, and therefore it seems umnecessary to waste ones effort at this point in producing dynamical models in a triaxial potential. This does not mean that, sooner or later, the potential of the galaxy will turn out to be triaxial, especially in the central regions. In fact, it is hard to see how it could not be! But, in any case, triaxiality in a tracer population (stars or gas) does not imply that the underlying gravitational potential is triaxial, since there is certainly no self-consistency requirement.

Similarly, since the radial velocity does not give any information on the $z$ component of the velocity for stars in the $\mathrm{GP}$, it is natural to try two integral models first (based on the specific binding energy $E$ and the $z$-component of the angular momentum, which are both integrals of the motion in an axisymmetric potential). Such models have the property that $\sigma_{r}=\sigma_{z}$. Only when the projected velocity dispersion turns out to be much too small (since for two-integral models $\sigma_{r}$ 

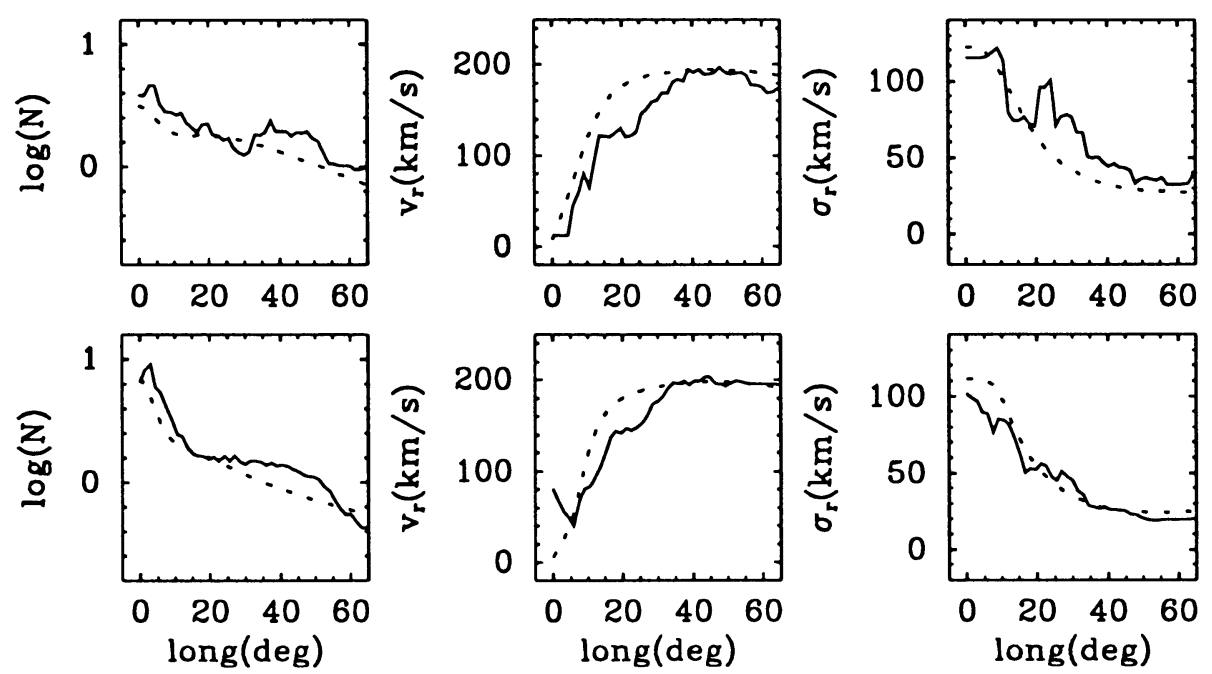

Fig. 3. The data for the tLH sample at $b=2^{\circ}$ (solid lines) compared with a 2-integral model (dashes) in an approximation to the BS potential. Left panels: logarithm of projected star counts per square degree, middle panels: projected mean velocity, right panels: projected velocity dispersions. Top panels: the old stars, bottom panels: the young stars.

is determined by the thinness of the disk), will we be able to rule out two-integral models.

Figure 3 shows the result of the fit of two-integral models embedded in an approximation to the Bahcall-Soneira potential (BS) for the Galaxy. For details, see te Lintel Hekkert et.al. (1991b). The data where smoothed by averaging at every point with the 15 closest neighbours, and the fit was produced on the basis of the projected star counts, fluxes and pressures. The fit looks good, to the degree that it cannot be expected to reproduce all details in the data, which may not be real anyway. Problem areas may be the projected velocity dispersion for the old stars, which is somewhat too low, and the mean velocity which rises a bit too fast for both samples. This may indicate the possible need for a third integral. In Fig.4, the spatial number density, mean rotation and radial velocity dispersion are plotted
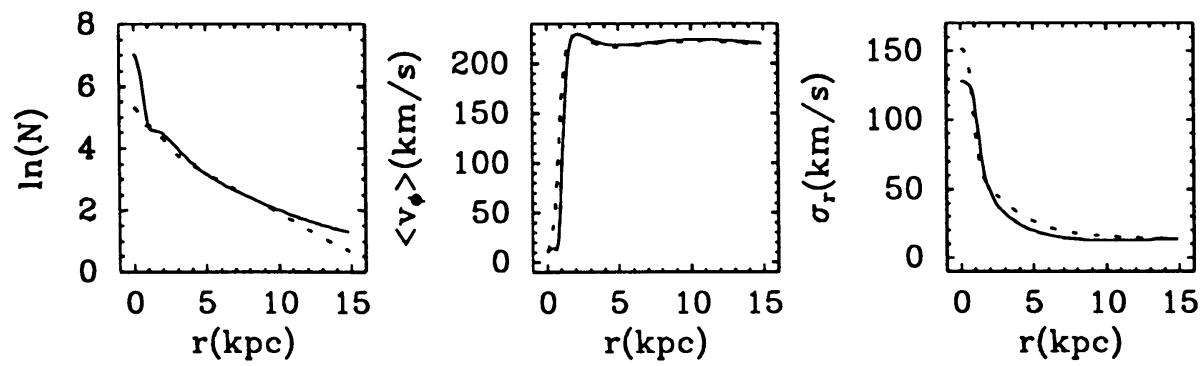

Fig. 4. The logarithm of the spatial density in the plane, the mean rotation and the radial velocity dispersion for the young (solid) and old (dashed) stars 
for both groups of stars. The old stars show a nice exponential disk (no artifact of the components used in QP!) with scale factor $3.5 \mathrm{kpc}$. The mean rotation follows very closely the rotation curve, and the old stars have overall a somewhat higher velocity dispersion, which clearly shows a bulge component. Finally, Fig. 5 shows the distribution function in turning point space (the color version is much nicer!). All well-known components are present. The dynamic range is very large: the highest value is about $10^{7} \mathrm{stars} / \mathrm{kpc}^{3} /(\mathrm{km} / \mathrm{s})^{3}$ in the thin disk, but, clearly, such values are very uncertain.

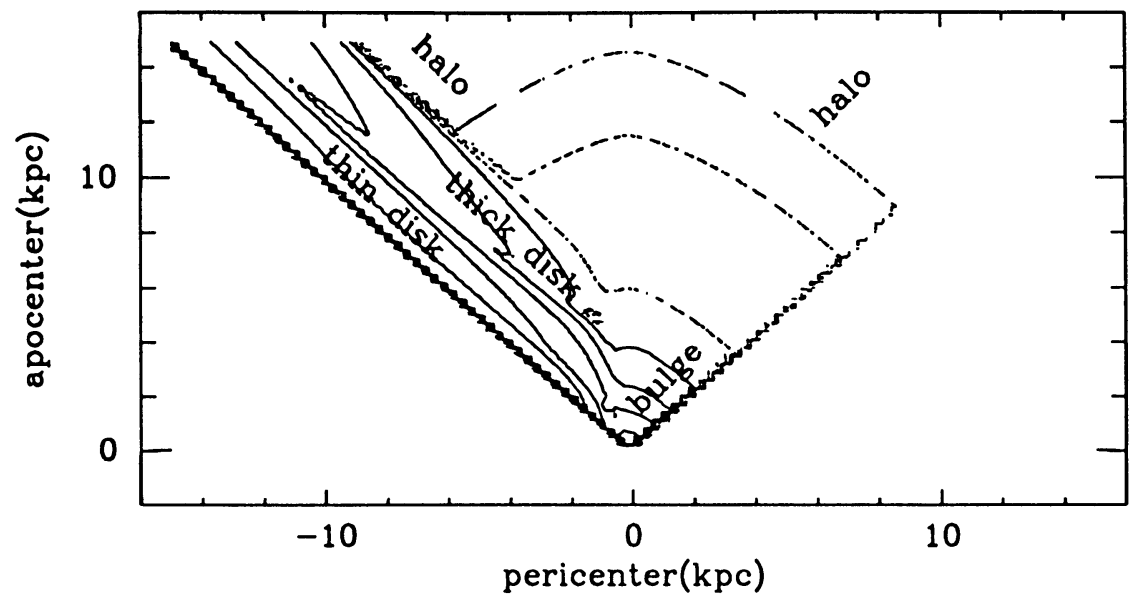

Fig. 5. The distribution function in turning point space. Contours are logarithmically spaced. Dotted contours correspond to values that are smaller than one. The dynamic range is of the order $10^{12}$.

\subsection{Planetary nebulae}

About 1500 PNs are known. An ancient but standard reference is the catalogue by Perek \& Kohoutek (1967). It has been (and still is) the basis for many surveys. This list however contains quite a few objects that later turned out to be something else, like M stars, symbiotic stars, or worse, HII regions. Subsequent cataloguing includes the work of Acker et.al. (1983), which is a good reference to the literature prior to 1983, and Acker et.al. (1991,1992).

A different approach uses the IRAS PSC, which, again, has been essential in the search for PN's. Just as is the case with the OH/IR stars, the PNs are strong infrared emittors, and occupy a fairly well defined place in the $F_{\nu}(12 \mu \mathrm{m}) / F_{\nu}(25 \mu \mathrm{m})$ versus $F_{\nu}(25 \mu \mathrm{m}) / F_{\nu}(60 \mu \mathrm{m})$ color diagram (Pottash et.al. 1988, Ratag et.al. 1990). Subsequent radio interferometry can be used to decide on the true nature of the candidates (see also Zijlstra et.al. 1989). This method up to now yielded about 50 new PNs within $15^{\circ}$ from the galactic center, on a total of about 400 in roughly the same region (Acker et.al. 1991). In any case, the final number of detected PNs in the Galaxy is in reasonable agreement with the total number of OH/IR stars known. 


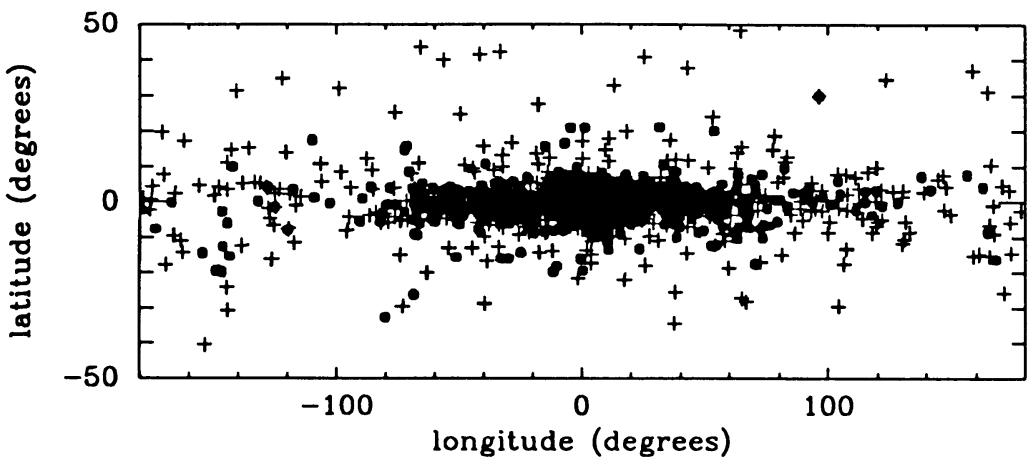

Fig. 6. The tlH sample (dots) ant the Acker et.al. sample (crosses)

No extensive dynamical modelling has been done as of now for the PNs. A preview of what is in store there can be seen by simply comparing the tLH sample and the Acker et.al. (1991) sample, such as if Fig. 6. The PN's extend towards higher latitudes then the OH/IR's. In the GP the coverage of the PNs is poor.

\section{Acknowledgements}

It is a pleasure to thank $\mathrm{H}$. Habing for providing the data of the OH/IR stars near the GC in electronic form, and A. Acker for doing the same for the PNs.

\section{References}

Acker, A., Marcout, J., Ochsenbein, F., Lortet, M.C., 1983, Astron. Astrophys. Suppl., 54, 315 Acker, A., Köppen, J., Stenholm, B., Raytchev, B., 1991, Astron. Astrophys. Suppl., 89, 237

Acker, A., Ochsenbein, Stenhohm, B., Tylenda, R., Marcout, J., Schohn, C., 1992, Strasbourg-ESO Catalogue of Galactic Planetary Nebulae, Part I, ESO publication

Dejonghe, H., 1989, Astrophys. J., 343, 113

Dejonghe, H. \& de Zeeuw, P.T., 1988, Astrophys. J., 329, 720

Dejonghe, H. \& Merritt, D., 1992, Astrophys. J.,

Eder, J., Lewis, B.M., Terzian, Y., 1988, Astrophys. J. Suppl., 66, 183

Goodman, J. \& Schwarzschild, M., 1981, Astrophys. J., 245, 1087

Habing, H.J., Olnon, F.M., Wimberg, A., Matthews, H.E., Baud, B., 1983, Astron. Astrophys., 128,230

Lindqvist, M., Wimberg, A., Habing, H.J., Matthews, H.E., 1992a, Astron. Astrophys. Suppl, 92, 43

Lindqvist, M., Habing, H.J., Wimberg, A., 1992b, Astron. Astrophys., 259, 118

Perek, L., \& Kohoutek, L., 1967, Catalogue of Galactic Planetary Nebulae, Prague Academic Press

Pottash, S.R., Bignell, C., Olling, R., Zijlstra, A.A., 1988, Astron. Astrophys., 205, 248

Ratag, M.A., Pottash, S.R., Zijlstra, A.A., Menzies, J., 1990, Astron. Astrophys., 233, 181

Sivagnanam, P., Braz, M.A., Le Squeren, A.M., Tran Minh, F., 1989, Astron. Astrophys., 211, 341.

te Lintel Hekkert, P., Caswell, J.L., Habing, H.J., Norris, R.P., Haynes, R.F., 1991a, Astron. Astrophys. Suppl., 90, 327

te Lintel Hekkert, P., Dejonghe, H., Habing, H.J., 1991b, Proc. of Astron. Soc. of Austr., 9, 20

van Langevelde, H.J., Brown, A.G.A., Lindqvist, M., Habing, H.J., de Zeeuw, P.T., 1992, Astron. Astrophys. Lett., submitted

Zijlstra, A.A., Pottash, S.R., Bignell, C., 1989, Astron. Astrophys. Suppl., 79, 329 